Rapport - Société canadienne d'histoire de l'Église catholique

\title{
Monseigneur Antoine Racine, premier évêque de Sherbrooke, en 1874 et la question universitaire canadienne
}

\section{Germain Lavallée}

Volume 32, 1965

URI : https://id.erudit.org/iderudit/1007330ar

DOI : https://doi.org/10.7202/1007330ar

Aller au sommaire du numéro

Éditeur(s)

Les Éditions Historia Ecclesiæ Catholicæ Canadensis Inc.

ISSN

0318-6148 (imprimé)

1927-7075 (numérique)

Découvrir la revue

Citer cet article

Lavallée, G. (1965). Monseigneur Antoine Racine, premier évêque de Sherbrooke, en 1874 et la question universitaire canadienne. Rapport - Société canadienne d'histoire de l'Église catholique, 32, 43-53.

https://doi.org/10.7202/1007330ar

Tous droits réservés @ Les Éditions Historia Ecclesiæ Catholicæ Canadensis Inc., 1966
Ce document est protégé par la loi sur le droit d'auteur. L'utilisation des services d'Érudit (y compris la reproduction) est assujettie à sa politique d'utilisation que vous pouvez consulter en ligne.

https://apropos.erudit.org/fr/usagers/politique-dutilisation/ 


\section{Monseigneur Antoine Racine premier évêque de Sherbrooke, en 1874 et la question universitaire canadienne}

La « question universitaire canadienne ", voilà comment on appelait le problème du dernier siècle relatif à la querelle longue, trop longue, qui s'est passée entre l'Université Laval de Québec et ce qui deviendra par la suite l'Université de Montréal.

Notre propos, aujourd'hui, ne sera pas de vous résumer l'ensemble de ce problème historique de la querelle. Nous restreignant à un aspect, non marginal cependant, mais intégrant de la question universitaire, nous allons vous présenter le rôle important qu'a joué Monseigneur Antoine Racine, premier évêque de Sherbrooke (et frère de Monseigneur Dominique Racine, évêque de Chicoutimi).

Tout d'abord, disons en gros ce qu'était « la question universitaire canadienne ».

Comme chacun sait, en 1852, le Séminaire de Québec avait obtenu une charte lui donnant droit d'ouvrir la première université catholique et canadienne-française de la province de Québec. Les dirigeants de la nouvelle université y invitèrent comme étudiants, non seulement les jeunes de la région de Québec, mais également les jeunes de Montréal et de toute la province.

Or il se trouva que les Montréalais, pour des raisons d'économie, de distance et, un peu, de chauvinisme, n'envoyaient pas leurs enfants à l'Université Laval. Ils crurent mieux faire de fréquenter McGill ou d'ouvrir à Montréal une école ou des écoles pour satisfaire aux besoins immédiats de la population de la région métropolitaine, qui dépassait déjà la population de la région de Québec.

Laval d'abord refuse toute affiliation à une école quelconque de Montréal, prétextant qu'elle est la seule université catholique, canadiennefrançaise du pays (ce que ne disait pas la charte, mais ce que dira plus tard un bill de l'assemblée législative de Québec), prétextant aussi que si Montréal obtenait une ou des écoles affiliées, elle finirait bien par obtenir une université comme à Québec, ce qui serait, croyait-on, la mort par asphyxie de Laval, privant cette dernière de professeurs, de finances et d'étudiants.

Devant ce refus de Laval, Mgr Bourget, archevêque de Montréal, prendra des positions fermes et agressives : $a$ ) les étudiants de Montréal n'iront jamais massivement à Québec, à cause de la grande distance qui sépare les deux villes, donc il faut' leur fournir sur les lieux les moyens de promotion universitaire; $b$ ) refuser aux catholiques de Montréal leur 
propre université, c'est les abandonner aux universités anglo-protestantes; de fait, après le refus de Laval, le docteur D'Orsonnens avait fait affilier son école de médecine à l'Université Victoria de Cobourg, en Ontario; et un nombre croissant d'étudiants catholiques fréquentaient McGill.

Voilà donc en résumé ce qu'on a appelé la "question universitaire canadienne", qui va faire couler des fleuves d'encre et accumuler des montagnes de papiers! Le seul petit travail de recherche que j'ai poursuivi m'a fait manipuler environ 2,000 documents écrits.

Cies documents, je les al puises aux archives de I'archevèche de Sherbrooke et aux archives du Séminaire de Québec et de l'Université Laval. En plus, la collection Jean-Baptiste Proulx (14, volumes), premier vice-recteur, Université Laval, à Montréal; pour retrouver le climat de l'époque, j'ai consulté Monseigneur Laflèche, de Rumilly, et Monseigneur Bourget, du même auteur.

Quel rôle a joué, dans la "question universitaire », Mgr Antoine Racine? Né aux Saint-Anges-Gardiens, près de Québec, instruit et formé au Petit et au Grand Séminaire de Québec, il était curé de la paroisse Saint-Jean-Baptiste de la ville de Québec quand, le 28 août 1874, il reçut de Pie IX la charge de fonder le diocèse de Sherbrooke. A l'époque, notre diocèse était, comme tous ceux de la province d'ailleurs, suffragant de l'archidiocèse de Québec. Et la question universitaire allait atteindre à sa plus grande effervescence.

Dès que fut connue la nomination du nouvel évêque, le problème ne mit pas de temps à se poser à Québec : Mgr Antoine Racine va-t-il être en faveur de l'Université Laval ou penchera-t-il plutôt vers Montréal et Mgr Bourget? Nos recherches nous ont amené à découvrir que le rôle de Mgr Racine a été sinon ambigu, du moins ambivalent.

\section{- I -}

Mgr T. E. Hamel, supérieur du Séminaire de Québec et recteur de Laval, écrit le 9 octobre 1874 à Mgr Benjamin Pâquet que « Mgr Racine est bien disposé à notre égard ». Mgr Pâquet n'en était pas pour autant très convaincu : "J'ai mis Monseigneur l'Archevêque [il s'agit de Mgr Taschereau ] en garde contre Mgr Racine qui est plus fourbe et plus traître que jamais. » Pourtant l'évêque de Sherbrooke écrira quelque temps après au recteur de Laval : "Espérons, avec M. le Recteur, que la Bulle Inter varias sollicitudines [de Léon XIII] (charte canonique) sera un signal de ralliement qui fera cesser les malentendus. Malentendus regrettables à l'extrême; malentendus qui devraient cesser et qu'on devrait faire cesser " (20 septembre 1876). Mgr Racine publie la Bulle dans son diocèse et encourage les jeunes étudiants du Séminaire à fréquenter, après leurs études secondaires, l'Université Laval. Désormais, tout le monde à Québec en est' satisfait. Une atmosphère de confiance va maintenant régner entre Québec et Sherbrooke. 
Mais, dès l'année suivante, les doutes vont ressurgir, lorsque Mgr Racine entreprend d'aller à Rome et qu'il fait très amicalement le voyage sur le même bateau qui transporte « les gens de Montréal » qui vont défendre la cause de leurs écoles, devant la Congrégation de la Propagande (à laquelle à l'époque étaient rattachées les universités). On s'inquiète de ces bons rapports. On accuse, à Québec, Mgr Racine d'être un rusé, voire un traître.

"Je suis convaincu que Mgr Racine nous fera du mal à Rome; je ferai mon possible pour parer les coups. Les siens seront d'autant plus terribles qu'il est regardé à Rome comme un grand ami de l'Université ", écrit Mgr Pâquet au Recteur de Laval, le 18 février 1877. Quels résultats Mgr Racine a-t-il vraiment obtenus à Rome dans ce voyage ? Rien pour, rien contre Laval, mais il a fait se poser la question de donner aux évêques suffragants (donc à ceux de la région de Montréal) un contrôle dans la nomination des professeurs et du recteur de l'Université (Mgr Pâquet à Mgr Taschereau, 3 juin 1877). Comme on peut voir, Mgr Racine était l'homme qui aimait se garder dans une certaine disponibilité.

En février 1876, l'Université Laval avait obtenu de Rome, par un décret de la Congrégation de la Propagande, le privilège de demeurer la seule université catholique de la province de Québec et le droit d'ouvrir une succursale à Montréal. Après bien des démarches, Mgr Conray, le Délégué apostolique, avait réussi à provoquer l'affiliation de l'école de médecine à la succursale montréalaise de l'Université Laval. Mais l'accord conclu ne dura guère. Montréal voulait garder toute son autonomie et n'entendait pas céder à Laval, se laisser conduire par les hommes de Québec. C'est pourquoi le docteur D'Orsonnens, directeur de l'école de médecine et de chirurgie de Montréal, décida de rompre avec Laval en alléguant l'illégalité de cette succursale, vu que la charte de Laval ne l'autorisait pas (au plan civil) à fonder une filiale hors de Québec et que le décret de Rome avait pourtant déclaré vouloir s'en tenir à la charte. La bataille reprend. Chaque camp envoie à Rome ses estafettes. L'Ecole Victoria de Montréal envoie en plus un délégué à Londres pour l'interprétation de la charte civile. Comme délégué à Rome, l'Archevêque de Québec choisit (en 1881) Mgr Antoine Racine, de Sherbrooke.

Cette fois, les Québecois vont en appeler au pape, en personne. Mgr Racine présente donc à Sa Sainteté Léon XIII, le 2 août 1881, un mémoire dont voici les principaux points :

$1^{\circ}$ Historique de l'Université Laval : cette demande d'université a été faite par tous les évêques de la province de Québec.

$2^{\circ}$ Le Séminaire de Québec n'a accepté de s'engager dans cette fondation qui exige des dépenses énormes, qu'à la condition que cette université demeure la seule de la province de Québec, tant que le nombre des catholiques ne suffirait pas pour soutenir deux universités.

$3^{\circ}$ Les gens de Montréal (y compris quelques évêques) n'ont pas voulu obéir au décret de Rome de 1877, par lequel Rome reconnaissait Laval comme devant être la seule université de la province de Québec. 
4. Travailler contre l'Université Laval c'est faire triompher le protestantisme au Canada. "Ils [les adversaires de Laval] prévinrent le ministre des Colonies à Londres, mettant en avant les intérêts protestants, pour l'engager à ne rien accorder à Laval. » (Le mémoire ne donne pas de preuves de cette accusation.)

Mgr Racine exige du Saint-Père une approbation solide, énergique et prompte pour la position de Laval et une condamnation non équivoque des adversaires, i.e. les évêques de Montréal et de Trois-Rivières,

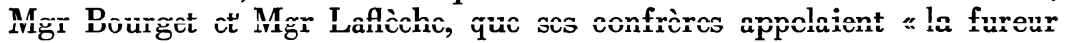
mitrée "!

Montréal ne laissera pas cependant les seuls Québecois renseigner les Congrégations et le Pape. En toute justice, avant de prendre position, Rome veut entendre Mgr Bourget et ses délégués. Mgr Racine est désappointé et craint que les choses ne traînent en longueur, affectant le progrès de Laval. Quelle fut la réponse du Saint-Père ? Allait-elle être aussi favorable à Laval qu'on le désirait chez les délégués de Québec ?

Pendant que Mgr Racine câblait à l'Archevêque de Québec que la zause de Laval était gagnée, l'abbé Dumesnil — un des délégués de Montréal - envoyait de son côté un télégramme en ces termes : «Confiance. Travaillons ferme.» Alors quoi ?

$1^{\circ}$ La thèse du docteur D'Orsonnens sur l'illégalité civile et canonique de la succursale n'était pas acceptée par Rome. Le Pape maintenait le décret de 1876, comme étant encore d'une valeur actuelle.

$2^{\circ}$ Laval doit rester la seule université tant que la population étudiante n'augmentera pas sensiblement et les Montréalais devront se contenter d'une succursale.

$3^{\circ}$ Les questions litigieuses devront être déférées au Conseil universitaire (de haute surveillance), c'est-à-dire à l'épiscopat de la province de Québec.

Décrets sur décrets et la lutte n'en est que plus engagée.

Nouveau décret de Rome en 1882, confirmant le décret de 1876 et de 1881. Le clergé de Montréal en est indisposé. Plus, quelques évêques commencent à sympathiser avec la cause des Montréalais, notamment Mgr Langevin, de Rimouski. Nouveau décret de Léon XIII, 27 février 1883, qu'on peut résumer en ces termes : comme l'Université Laval et sa succursale établie à Montréal par l'autorité apostolique se trouvent depuis longtemps en butte à de grandes difficultés à cause des discussions qu'on a soulevées, le Saint-Père demande : $1^{\circ}$ qu'on s'en tienne au décret de $1876 ; 2^{\circ}$ à la constitution, i.e. à la charte civile; $3^{\circ}$ de ne pas tramer quoique ce soit contre l'Université Laval; $4^{\circ}$ de faire tout le possible pour son expansion.

Ici une remarque : Mgr Ignace Bourget avait démissionné motu proprio en 1881, et avait comme coadjuteur, Mgr Fabre, un homme serein, affable et doux. Les Montréalais n'en continueront pas moins à 
ouvrir les portes de l'Ecole Victoria, contre le gré de Laval et... des décrets. L'Archevêque de Québec menace d'excommunication professeurs et élèves de l'Ecole. Rome s'émeut : "Suspende omnia » disait un télégramme adressé par le cardinal Siméoni à l'Archevêque de Québec (août 1883). A Québec on s'énerve. On parle de fermer les portes de Laval en septembre. Une grève, quoi !

Mgr Racine invite les Québecois au calme :

"Quant à fermer à l'automne les cours à Québec et à Montréal, je suis loin de cet avis. Le St-Siège n'aura pas cette mesure extrême pour agréable. D'ailleurs il sera toujours temps de faire cela, lorsque le Commissaire (délégué apostolique) sera sur les lieux et qu'il aura été prévenu de votre détermination. »

Le "suspende omnia " de Rome releva le courage des Montréalais : non seulement, ils veulent une faculté de médecine, mais "les avocats veulent aussi avoir une faculté de droit indépendante ».

Malheureusement, le délégué apostolique, le T.R.P. Smeulders ne règlera rien. Aigreur de part et d'autre.

L'aigreur de l'époque va pourtant être adoucie par un peu de miel ... Et quand Rome accorde du miel, ce n'est pas toujours pour la seule flatterie du palais; c'est quelque fois pour faire avaler la médecine ! En 1886, l'Ârchevêque de Québec est élevé au Cardinalat.

Les Evêques de Montréal et d'Ottawa deviennent Archevêques.

Le Grand Vicaire Hamel (de Québec) reçoit le titre de Protonotaire Apostolique et l'Evêque de Sherbrooke, Assistant au trône pontifical. Mais, notez bien : le diocèse de Sherbrooke avec son titulaire Mgr Racine, défenseur de Laval à Rome, devient suffragant de Montréal. Mgr Fabre invite son suffragant à venir discuter à Montréal des grands problèmes de l'heure.

1886, peut être considérée comme la table tournante de la question universitaire, du moins pour le rôle qu'y joue l'Evêque de Sherbrooke.

II

Pour emprunter le style de l'abbé Proulx (V.-R. à Montréal) disons que les honneurs ecclésiastiques avaient charrié dans leur gorgée sucrée, la division du territoire catholique du Canada français en trois archidiocèses : Québec, Montréal, Ottawa. Quelques trois ans après - le 2 février 1889 - sa Sainteté Léon XIII adressait une lettre aux Evêques du Canada français dans laquelle il demandait, non à tous, mais spécifiquement aux Evêques de la Province ecclésiastique de Montréal de régler le problème de la Succursale de l'Université Laval à Montréal.

C'est la fameuse lettre "Jam dudum». Le travail de pacification était d'ores et déjà amorcé depuis 1887. Le Séminaire de Montréal demandait qu'on organisât la Faculté des Arts de la Succursale. L'Ecole Polytechnique veut passer sous le contrôle de la Succursale. On parle de plus en plus de l'Université à Montréal et non de la Succursale. On 
parlera bientôt de l'Université de Montréal. Mgr Racine fait justement remarquer, et cela, avant même la lettre de Léon XIII (Jam dudum) : "Dans le bill proposé » "Acte pour incorporer le Syndicat", je remarque avec bonheur que le mot "Succursale " n'est pas mentionné, et c'est très bien fait. Je vous dirai en toute franchise qu'il faut éviter d'employer inutilement, un mot aussi impopulaire, qui sonne si mal aux oreilles des vieilles Montréalaises... Ce mot nuisait à la bonne entente et ne servirait qu'à entretenir les préjugés qui existent déjà. » Il ajoute :

«Quant au paragraphe (a) dont je vous ai parlé déjà, il vaut mieux ne pas nommer Gouverneurs de droit, les Evêques de St-Hyacinthe et de Sherbrooke et laisser de côté aussi les futurs Evêques suffragants, parmi lesquels pourraient fort bien se trouver quelques-uns des adversaires de Laval. »

(Mgr A. Racine à l'abbé Marcoux Vice-Recteur de Laval à Montréal)

Par où l'on voit un effort pour que soient supprimées toutes les causes de frictions. Dans sa lettre "Jam dudum », Léon XIII avait confié aux Evêques de la Province Ecclésiastique de Montréal, la nomination du Vice-Recteur de Laval à Montréal (fonction qui équivalait à Recteur à Montréal). L'Evêque de Sherbrooke est consulté par le Premier Ministre et par les Administrateurs de Montréal. A ce moment, la candidature semblait favoriser l'abbé Bruchési. L'honorable J. O. Chauveau demande à Mgr A. Racine d'y mettre son véto : " Je crois qu'il serait très prudent de ne pas vous engager pour cette nomination. Il y a beaucoup d'objections. Je suppose que d'autres vous auront écrit dans ce sens. " (21 mars 1889)

Le même jour, le Vice-Recteur qui doit démissionner, annonce à l'Evêque de Sherbrooke : "Je me propose d'aller vous rendre visite le plus tôt possible pour discuter avec votre Grandeur la situation présente et prendre vos conseils » (21 mars 1889).

Quelques semaines plus tard, on apprend que le nouveau ViceRecteur n'est pas l'abbé Bruchési, mais le curé de Saint-Lin des Laurentides, l'abbé Jean-Baptiste Proulx. Chacun s'en félicite. Mgr Racine y a été pour quelque chose.

Un problème urgent se pose, l'union des deux écoles de Médecine, l'Ecole Victoria et l'Ecole de Laval. Mgr Pâquet de Laval hésite, car il n'a pas confiance en l'Ecole Victoria et croit que les élèves de Laval à Montréal subiront par cette union, une baisse dans le niveau des études. Cependant Mgr Racine travaille à l'Union, appuyée d'ailleurs en cela par le Cardinal Siméoni qui écrit aux Evêques de la Province Ecclésiastique de Montréal le 12 juin 1890 : " qu'on s'entende de part et d'autre pour réaliser l'union et pour accepter de grand cœur les sacrifices exigés par une œuvre si utile. » 
Mgr Racine est d'avis que les trois évêques de la Province Ecclésiastique de Montréal écrivent une lettre circulaire commune, par laquelle ils donneraient à tous leurs diocésains, connaissance :

$1^{\circ}$ de la lettre du Saint-Siège à l'Archevêque de Montréal et à ses Suffragants;

$2^{\circ}$ du document qu'ils venaient de signer, par lequel un comité était nommé pour s'entendre avec l'Ecole de Médecine do Montréal;

$3^{\circ}$ du désir d'obéir aux volontés du Saint-Siège, et pour cela faire appel à tous les gens de bien, qui aiment leur religion et leur pays.

(Mgr Racine à L.-D.-A. Maréchal,

3 septembre 1890)

La lettre circulaire va paraître le 8 novembre 1890 et dans les termes suggérés par Mgr Racine. Le comité fut composé et se mit à discuter un projet d'union qui comprenait deux closes :

$1^{\circ}$ L'Ecole de Médecine devient la Faculté de Médecine.

$2^{\circ}$ Les biens possédés par l'ancienne Ecole restent la propriété des membres de cette école qui peuvent se les diviser entre eux ou les passer à la nouvelle Faculté de Médecine de l'Université.

Le comité fait l'accord sur ces deux points. Mgr de Sherbrooke se rend ensuite à Montréal pour arbitrer la rencontre des deux comités (celui de la Succursale et celui de l'Ecole Victoria). "Les médecins de l'Ecole (Victoria) ont été satisfaits de notre entrevue d'hier soir, écrit l'abbé Proulx à Mgr Racine. Vous les avez gagnés tout à fait par vos manières de faire, franches et larges. *

On demande alors au Gouvernement de Québec de sanctionner l'Union par un bill. Mercier, qui est alors premier ministre, craint d'y jouer de son capital politique à Québec. Il exige, avant de procéder que Rome donne un ordre exprès. Double télégramme à Rome pour presser les affaires. Mgr Fabre qui est lui-même à Rome pour régler la question, insiste auprès de Mgr Siméoni. Tout aurait marcher, mais on avait oublié à Montréal qu'on ne pouvait pas s'entendre ainsi à l'amiable sans la permission du Conseil Universitaire de Laval !

"L'Université Laval... est grandement surprise et peinée de voir que ... le Conseil Universitaire n'ait pas été prévenu et n'ait pas eu l'occasion de donner son avis.»

(Mgr TAGhereau à Mgr Racine, 22 novembre 1890.)

L'Evêque de Sherbrooke écrit là-dessus, à l'abbé Proulx : « Cependant il faut marcher de l'avant...., il ne faudra point, pour aucune raison, laisser entamer les points fondamentaux de l'Union, tels qu'exprimés dans le Bill.» (17 novembre 1890.) 
Le 20 novembre, Rome câble au Premier Ministre de sanctionner le bill. Le bill fut donc présenté à la Chambre le même jour, puis en deuxième lecture le 23 novembre et devra ensuite subir l'épreuve des bills privés : "C'est le passage de la mer Rouge.»

(L'abbé Proulx à Mgr Racine, 21 novembre.)

L'Evêque de Sherbrooke répond au Vice-Recteur dans le même style humoristique : " Je vous souhaite un bon passage de la Mer Rouge aussi heureux que celui de Moïse; tout de même soyez sur vos gardes, car l'armée de Pharaon vous suit de près. \$ (23 novembre.)

L'armée de Pharaon, c'était de toute évidence le Conseil Universitaire de Laval ! Le bill passa en $3^{\circ}$ lecture le 29 novembre 1890 . Comme on pouvait s'y attendre, la chose blessa profondément les administrateurs de l'Université de Québec. Les difficultés n'étaient pas encore terminées.

Un dernier problème cependant mêlera Mgr Racine à la question universitaire : le syndicat financier de l'Université de Montréal. Il fallait pourvoir l'Université de Montréal d'un Conseil d'affaires. La suggestion en était venu de l'Evêque de Sherbrooke.

(Proulx à Racine, 28 février 1891.)

A Montréal, les choses allaient d'elles-mêmes. La paix était faite. Laval y voit des objections majeures (le Cardinal Tachereau à l'abbé Proulx) :

"Sous prétexte de faire incorporer civilement (les administrateurs de l'Université Laval à Montréal, le projet de loi que vous présentez à l'approbation du Conseil Supérieur de l'Université (sans nous dire ce qu'en pense le Saint-Siège), crée réellement à Montréal, une Université tout à fait indépendante de l'Université Laval telle que constituée par sa Charte Royale et son institution canonique.

Dans ces conditions, je ne puis pour ma part, en ma qualité de Chancelier Apostolique de l'Université Laval, approuver ce projet de loi, et je ne l'appuierai qu'autant qu'il me sera authentiquement démontré que telle est la volonté du Saint-Siège. "

Mgr Racine a tout compris, quand il eut pris connaissance de ce document. Le Cardinal va en appeler à Rome. Voici donc quelles suggestions, L'Evêque de Sherbrooke donne à l'abbé Proulx, pour sortir de l'impasse :

$1^{\circ}$ demander à tous les Evêques de la Province de Québec leur opinion par écrit et sans délai. Puisque le Cardinal a déjà déclaré qu'il désapprouvait le projet du bill, " c'est le seul moyen que nous avons de faire connaître au Saint-Siège l'opinion et les sentiments des Evêques sur cette question ";

$2^{\circ}$ Son Eminence le Cardinal Taschereau a choisi le terrain de la lutte : Rome. Impossible de reculer. «C'est à Mgr l'Arche- 
vêque de Montréal qu'il appartient de choisir celui qui doit aller défendre, à Rome, les intérêts si graves de l'Université Laval à Montréal. Dans mon humble opinion, il n'y a pas de temps à perdre. 》 Qui sera le défenseur des droits de Montréal contre les prétentions de Laval, à Rome? Les besoins de la cause peuvent faire naître ici un drôlatique paradoxe...

\section{Le voyage à Rome de Mgr A. Racine en 1891}

Mgr Racine est de plus en plus gagné à la cause de l'Université à Montréal : la région de Montréal augmentait sans cesse de population; le diocèse de Sherbrooke était devenu le suffragant de Montréal, la lettre du Saint-Père de 1889 demandant aux Evêques de la Province Ecclésiastique de Montréal de régler la question universitaire, la personnalité plus attachante de Mgr Fabre, voilà autant de raisons qui ont engagé l'Evêque de Sherbrooke dans la cause des Montréalais. Aussi, n'est-on pas trop étonné de voir que Mgr Fabre, pour s'assurer à Rome l'influence d'un homme très au fait de la question universitaire, choisisse l'Evêque de Sherbrooke.

Mgr Racine accepte cette mission d'aller prouver que l'Archevêque de Montréal, ses suffragants et le vice-recteur ont tout fait ce que le SaintPère a demandé pour l'Union des Ecoles à Montréal et qu'ils ont réussi. Il ne reste plus qu'à obtenir le «Syndicat financier \$ que veut refuser Laval.

L'argument que Mgr Racine va brandir pour gagner sa cause : si Rome ne nous appuie pas, Laval va nous enlever l'autonomie que nous avons déjà, les professeurs vont démissionner plutôt que de se soumettre à un Vice-Recteur de Laval et ce sera abandonner les jeunes étudiants catholiques à l'Université protestante, voire à la fondation d'une université neutre.

A cette étape de la question, les Administrateurs de Laval à Québec décident de faire la grève du silence : deux demandes par télégramme sont envoyées au Cardinal Taschereau par le Cardinal Siméoni. Aucune réponse. Laval espère ainsi gagner du temps et faire en sorte que le bill du « syndicat financier » ne puisse pas être présenté avant la fin de la session du Parlement de Québec qui doit s'ajourner le 7 avril 1892. Par malheur survient aussi la mort subite du cardinal Siméoni, bien au fait de toutes ces questions et plutôt favorable à la position des Montréalais. Mais coup sur coup l'Evêque de Sherbrooke - qui est toujours à Rome - adresse un mémoire à la Congrégation de la Propagande et un autre au Saint-Père lui-même, où il montre qu'un retard à faire sanctionner le bill du Syndicat financier peut amener la catastrophe, i.e. l'écrasement d'une Université Catholique à Montréal et la fondation d'une université française à caractère nettement religieux.

Rome exige certains compromis pour éviter de choquer Laval, en rapport avec la nomination du Vice-Recteur et la fondation de nouvelles facultés. Il sera imposé à Montréal de consulter le Conseil universitaire de Laval. Les gens de Montréal, pilotés par Mgr Racine, 
acceptent ces conditions. Rome veut encore dissiper un malentendu : les cours de l'Ecole Victoria valent-ils les cours de la Succursale ? Mgr Racine contredit les renseignements fautifs que Mgr Pâquet avait donnés à la Congrégation, à l'effet que les cours de l'Ecole Victoria n'étaient que de deux ans et donc inférieurs aux cours de la succursale qui étaient de quatre ans.

« Nous regrettons d'avoir à le dire, ces avancés sont le contraire de la vérité : les cours de l'Ecole de Médecine et de Chirurgie de Montréal sont, depuis nombre d'années de quatre ans. C'est un fait notoire et public qui n'a pas besoin d'êire prouvé. »

(Faut dire, en critique, que cependant les années de l'Ecole Victoria étaient de six mois; celles de la Succursale de neuf mois. Ce que Mgr Racine semble avoir oublié de mentionner dans sa réponse à la question de la Congrégation).

Montréal triomphait. Aussi Monsieur Colin p.s.s. écrit-il à Mgr Racine : "Jamais le pays ne saura assez reconnaître ce dont il vous sera redevable. *

Et Mgr Fabre : " Monseigneur, dans toute cette campagne [car on était en guerre !] Votre Grandeur a travaillé énergiquement pour le bien de la Province de Montréal. Tous devront vous en être reconnaissants mais personne plus que le Métropolitain." Laval est vaincu. Le «Syndicat financier» sera. Mgr Pâquet songe alors à une forme de représailles très efficace : demander aux Evêques de la Province civile de Québec une indemnité pour les dépenses que le Séminaire a faites pour l'Université à Montréal. Mgr Racine écrit un nouveau mémoire au préfet de la Congrégation de la Propagande, pour réfuter les prétentions de Mgr Pâquet. Autre difficulté : de retour à Montréal, Mgr Racine apprend que les Montréalais sont eux-mêmes marris que les deux délégués à Rome aient consenti à des compromis. On menace de refuser de présenter le bill à la Législature. Négociations à Montréal avec les administrateurs de la Succursale, négociations avec le Supérieur du Séminaire de Québec : le bill finit' par être soumis à la Législature. L'Université de Montréal désormais pourvu d'un "Syndicat financier.» va maintenant commencer sa lente marche, d'abord aidée par les Sulpiciens qui lui font un don de $\$ 50,000$. et d'un terrain. L'Evêque de Sherbrooke demande aux Administrateurs de l'Université de Montréal de tenir au courant le Saint-Siège, des nouveaux développements. \& Le mémoire sera rédigé par l'abbé Proulx, V.-R., dans le sens que vous désirez, Mgr. »

Les plans de l'Université (de la rue St-Denis) sont terminés, les soumissions sont demandées, le 6 juillet 1893. L'Evêque de Sherbrooke s'en réjouit :

" J'ai bien hâte de vous voir et de parler de toutes les choses qui nous intéressent à un si haut degré : notre Université, la question de l'éducation... Je vous attendrai donc le 25 juillet jusqu'au 30 juillet, car je dois m'absenter le 31 , pour deux semaines. »

Cette entrevue désirée n'eut pas lieu. Mgr Racine allait s'absenter ... pour toujours. Le 17 juillet, après quelques jours de maladie, 
il expirait dans son évêché. C'est l'abbé Proulx, vice-recteur de l'Université de Montréal, qui fit son éloge funèbre.

\section{CONCLUSION}

Le rôle de Mgr Antoine Racine dans la question universitaire en a été un de tout premier plan. Il a été par sa sagesse et la prudence de ses conseils, l'âme dirigeante d'une époque de transition. Son influence auprès des dirigeants de l'Université Laval d'abord, son amitié de plus en plus solide pour les administrateurs de l'Université de Montréal ensuite, surtout depuis 1886, et un rôle tout de diplomatie auprès des congrégations romaines ont fait de lui une des belles figures de l'époque.

Germain LAVALLÉE, ptre.

Directeur des études au Séminaire de Sherbrooke.

N.B. - Cette conférence est un résumé d'un travail scientifique de 100 pages, dont l'auteur peut offrir encore quelques exemplaires. 